\title{
Scheduling Simple Linear Deteriorating Jobs with Rejection
}

\author{
Juan Zou ${ }^{1,2}$ and Yuzhong Zhang ${ }^{1}$ \\ ${ }^{1}$ School of Management, Qufu Normal University, Rizhao, Shandong 276826, China \\ ${ }^{2}$ School of Mathematical Sciences, Qufu Normal University, Qufu, Shandong 273165, China
}

Correspondence should be addressed to Juan Zou; zhoujuan.zj12@gmail.com

Received 28 March 2014; Accepted 31 May 2014; Published 14 July 2014

Academic Editor: Guoqiang Zhang

Copyright (C) 2014 J. Zou and Y. Zhang. This is an open access article distributed under the Creative Commons Attribution License, which permits unrestricted use, distribution, and reproduction in any medium, provided the original work is properly cited.

\begin{abstract}
We consider the problems of scheduling deteriorating jobs with release dates on a single machine (parallel machines) and jobs can be rejected by paying penalties. The processing time of a job is a simple linear increasing function of its starting time. For a single machine model, the objective is to minimize the maximum lateness of the accepted jobs plus the total penalty of the rejected jobs. We show that the problem is NP-hard in the strong sense and presents a fully polynomial time approximation scheme to solve it when all jobs have agreeable release dates and due dates. For parallel-machine model, the objective is to minimize the maximum delivery completion time of the accepted jobs plus the total penalty of the rejected jobs. When the jobs have identical release dates, we first propose a fully polynomial time approximation scheme to solve it. Then, we present a heuristic algorithm for the case where all jobs have to be accepted and evaluate its efficiency by computational experiments.
\end{abstract}

\section{Introduction}

For most classical scheduling problems, the processing times of jobs are considered to be constant and independent of their starting time. However, this assumption is not appropriate for the modelling of many modern industrial processes; we often encounter situations in which processing time increases over time, when the machines gradually lose efficiency. Such problems are generally known as scheduling with deterioration effects. Scheduling with linear deterioration was first considered by Browne and Yechiali [1] who assumed that the processing times of jobs are nondecreasing and start time dependent linear functions. They derived optimal scheduling policies that minimize the expected makespan. Mosheiov [2] considered simple linear deterioration where jobs have a fixed job-dependent growth rate but no basic processing time. He showed that most commonly applied performance criteria, such as the makespan, the total flow time, the total lateness, the sum of weighted completion time, the maximum lateness, the maximum tardiness, and the number of tardy jobs, remain polynomially solvable. Since then, machine scheduling problems with deteriorating jobs have received increasing attention. An extensive survey of different models and problems was provided by Alidaee and Womer [3].
Cheng et al. [4] presented an updated survey of the results on scheduling problems with time-dependent processing times. Other new results of scheduling with deterioration effect can be found in [5-7].

At the same time, it is always assumed in traditional research that all the jobs have to be processed. In the real world, however, things may be more complicated. For example, due to limited resources or limited available capacity, the decider of a factory can choose only a subset of these tasks to be scheduled, while perhaps incurring some penalty for the rejected jobs. The machine scheduling problem with rejection was first introduced by Bartal et al. [8]. They studied both the offline and online versions of scheduling problem with rejection on identical parallel machines. The objective is to minimize the sum of the makespan of the accepted jobs and the total rejection penalty of the rejected jobs. After that, the machine scheduling problem with rejection received more and more attention. Hoogeveen et al. [9] considered the offline multiprocessor scheduling problem with rejection where preemption is allowed. Zhang et al. [10] studied the single machine scheduling with release dates and rejection to minimize the sum of the maximum completion times of the scheduled jobs and the total penalty of the rejected jobs. Sengupta [11] considered algorithms and approximation 
schemes for minimum lateness/tardiness scheduling with rejection. Shabtay et al. [12] recently presented an updated survey on offline scheduling with rejection. In addition, there has been some work on scheduling deteriorating jobs with rejection. For example, Cheng and Sun [13] considered the single machine scheduling problem with deterioration and rejection, in which the processing time of a job is a linear function of its starting time. Li and Yuan [14] studied several parallel-machine scheduling problems in which the processing time of a job is a linear increasing function of its starting time and jobs can be rejected by paying penalties. The objectives are to minimize the makespan and the total weighted completion time plus the total penalty of the rejected jobs. Hsua and Chang [15] considered the unrelated parallel-machine scheduling with deteriorating jobs and rejection. To the best of our knowledge, the problems of scheduling deteriorating jobs with release dates and rejection have not been discussed.

In this paper, we study the single machine (parallelmachine) scheduling of deteriorating jobs with release dates and rejection. The paper is organized as follows. In Section 2, we formulate the problems under consideration and introduce some notations. In Section 3, we first discuss single machine model and show that the problem is strongly NPhard. Then we propose a fully polynomial time approximation scheme for the case where all jobs have agreeable release dates and due dates. In Section 4, we consider parallelmachine scheduling problem with deterioration and rejection. In Section 5, we conclude the paper and suggest some topics for future research.

\section{Problem Description and Notation}

The problems considered in this paper can be formally described as follows. There are an independent job set $\mathscr{J}=$ $\left\{J_{1}, \ldots, J_{n}\right\}$ and a single machine $(m \geq 2$ identical parallel machines $\left.\left\{M_{1}, \ldots, M_{m}\right\}\right)$. Each job $J_{j}(j=1, \ldots, n)$ has a release date $r_{j}$, a due date $d_{j}$ (a delivery time $q_{j}$ ), a deteriorating rate $b_{j}>0$, and a rejection penalty $e_{j}$. Each job $J_{j}(j=1, \ldots, n)$ is either rejected with a rejection penalty $e_{j}$ having to be paid or accepted and processed on the single machine (one of parallel machines). The actual processing time of job $J_{j}$ is given by $p_{j}=b_{j} t$, where $t \geq r_{j}$ is the starting time of $J_{j}$. Denote by $R$ and $\bar{R}=\mathscr{J} \backslash R$ the set of rejected jobs and the set of accepted jobs, respectively. Denote by $C_{j}$ the completion time of the accepted job $J_{j}$. In the single machine model, assume $\min \left\{r_{j} \mid j=1, \ldots, n\right\} \geq t_{0}>0$; the lateness of job $J_{j}$ is defined as $L_{j}=C_{j}-d_{j}$. The objective is to minimize the maximum lateness (i.e., $L_{\max }=\max \left\{L_{j}: J_{j} \in \bar{R}\right\}$ ) plus the total penalty of the rejected jobs. In parallel-machine model, assume $\min \left\{r_{j} \mid j=1, \ldots, n\right\}=t_{0}>0$. We consider that each job $J_{j}$ has a delivery time $q_{j} \geq 0$ rather than a due date $d_{j}$. The delivery of a job begins immediately after it completes processing, and job deliveries are allowed to overlap. We define the delivery completion time as $D_{j}=$ $C_{j}+q_{j}$; the objective is to minimize the maximum delivery completion time (i.e., $D_{\max }=\max \left\{D_{j}: J_{j} \in \bar{R}\right\}$ ) plus the total penalty of the rejected jobs. Using the three-field notation of Graham et al. [16], the problems are denoted by $1 \mid r_{j}, p_{j}=b_{j} t$, rej $\mid L_{\max }(\bar{R})+\sum_{J_{j} \in R} e_{j}$ and $P m \mid r_{j}=t_{0}, p_{j}=$ $b_{j} t$, rej $\mid D_{\max }(\bar{R})+\sum_{J_{j} \in R} e_{j}$.

\section{The Single Machine Problem}

3.1. NP-Hardness Proof. In this subsection, we first discuss the complexity of the problem $1\left|r_{j}, p_{j}=b_{j} t\right| L_{\max }$ where rejection is not considered.

Theorem 1. The problem $1\left|r_{j}, p_{j}=b_{j} t\right| L_{\max }$ is strongly NP-hard.

Proof. The decision version of the scheduling problem is clearly in NP. We use a reduction from the 4-product problem (4-P), which is strongly NP-hard (see [17]).

An instance of the 4-product problem is formulated as follows. Given $D \in Q^{+}$, a finite set $N=\{1,2, \ldots, 4 k\}$ for some natural number $k$, a size $y_{j} \in Q^{+}$and $D^{1 / 5}<y_{j}<D^{1 / 3}$ for every $j \in N, \prod_{j \in N} y_{j}=D^{k}$. Does there exist disjoint subsets $N_{1}, \ldots, N_{k}$ such that $\bigcup_{i=1}^{k} N_{i}=N$ and the product of sizes of the elements in $N_{i}$ satisfies $\prod_{j \in N_{i}} y_{j}=D$ for $1 \leq i \leq k$ ?

Given an arbitrary instance I of 4 -product problem, we construct an instance II of the scheduling problem as follows.

(i) There are $n=5 k-1$ jobs.

(ii) For $1 \leq j \leq 4 k, r_{j}=1, b_{j}=y_{j}-1$, and $d_{j}=2^{k-1} D^{k}$.

(iii) For $4 k+1 \leq j \leq 5 k-1, r_{j}=2^{j-4 k+1} D^{j-4 k}, b_{j}=1$, and $d_{j}=2^{j-4 k} D^{j-4 k}$.

(iv) The threshold value is defined by $Y=0$.

The decision version asks whether there is a schedule $\pi$ for problem $1\left|r_{j}, p_{j}=b_{j} t\right| L_{\max }$ such that $L_{\max } \leq Y$.

It is clear that the reduction can be done in polynomial time. Now we prove that instance I has a solution if and only if instance II has a schedule $\pi$ with $L_{\max } \leq Y$.

Assume that 4-product problem has a solution; that is, there exist disjoint subsets $N_{1}, \ldots, N_{k}$ such that $\bigcup_{i=1}^{k} N_{i}=$ $N$ and the product of sizes of the elements in $N_{i}$ satisfies $\prod_{j \in N_{i}} y_{j}=D$ for $1 \leq i \leq k$. For convenience, we denote the set of jobs $\left\{J_{j}: j \in N_{l}\right\} \quad(1 \leq l \leq k)$ by $J_{N_{l}}$. The jobs are scheduled without idle times as follows. First, the set of jobs $J_{N_{l}}$ are scheduled in the interval $\left[2^{l-1} D^{l-1}, 2^{l-1} D^{l}\right]$ for $l=1, \ldots, k$. Next, each job $J_{j}$ is scheduled at time $r_{j}=2^{j-4 k+1} D^{j-4 k}$ for $j=4 k+1, \ldots, 5 k-1$. Therefore, the maximum lateness of this schedule is $L_{\max }=\max \left\{C_{j}-d_{j}: 1 \leq j \leq 5 k-1\right\}=0$.

Conversely, assume that there is a schedule $\pi$ with $L_{\max } \leq$ 0 . We are ready to prove that instance I has a solution.

First, each job $J_{j}(4 k+1 \leq j \leq 5 k-1)$ must start exactly at its release date $r_{j}$ and finish at its due date $d_{j}$, since $L_{\max } \leq$ $Y=0$. Second, the rest $4 k$ jobs are scheduled as the $k$ intervals from time $t=1$ to time $t=2^{k-1} D^{k}$. Without loss of generality, let $J_{N_{l}}(1 \leq l \leq k)$ be the set of jobs scheduled in the interval $\left[2^{l-1} D^{l-1}, 2^{l-1} D^{l}\right]$. 
Therefore, from the above discussion, we know that $\prod_{j \in N_{l}} y_{j} \leq D$ for $1 \leq l \leq k$. Note that $\prod_{l=1}^{k} \prod_{j \in N_{l}} y_{j}=$ $\prod_{j=1}^{4 k} y_{j}=D^{k}$. Thus, we have $\prod_{j \in N_{l}} y_{j}=D$ for $1 \leq l \leq k$. That is, instance I has a solution. This completes the proof.

Corollary 2. The problem $1 \mid r_{j}, p_{j}=b_{j} t$, rej $\mid L_{\max }(\bar{R})+$ $\sum_{J_{j} \in R} e_{j}$ is strongly NP-hard, so it has no fully polynomial time approximation scheme unless $P=N P$.

3.2. An FPTAS for One Special Case. An algorithm $\mathscr{A}$ is a $\rho$ approximation $(\rho \geq 1)$ algorithm for a minimization problem if it produces a solution that is at most $\rho$ times than the optimal one ( $\rho$ is also referred to as the worst-case ratio). A family of algorithms $\left\{\mathscr{A}_{\epsilon}: \epsilon>0\right\}$ is called a fully polynomial time approximation scheme (FPTAS), if for each $\epsilon>0$, the algorithm $\mathscr{A}_{\epsilon}$ is a $(1+\epsilon)$ approximation algorithm running in polynomial time in the input size and $1 / \epsilon$.

In this subsection, we consider the special case where all jobs have agreeable release dates and due dates and assume that the jobs have been indexed such that $r_{1} \leq \cdots \leq r_{n}$ and $d_{1} \leq \cdots \leq d_{n}$. The problem can be denoted by $1 \mid r_{j}, p_{j}=$ $b_{j} t$, rej, agreeable $\mid L_{\max }(\bar{R})+\sum_{J_{j} \in R} e_{j}$.

We design an FPTAS for this problem by considering the modified deteriorating rates, the modified release dates, the modified due dates, and the inflated rejection penalty. The definition of the modified deteriorating rates and the modified release dates involves a geometric rounding technique developed by Afrati et al. [18]. The definition of the inflated rejection penalty is stated by Sengupta [11]. The rounding technique and the inflated rejection penalty are stated, respectively, as follows.

For any $\epsilon^{\prime}>0$ and $x \geq 1$, if $\left(1+\epsilon^{\prime}\right)^{k-1}<x<\left(1+\epsilon^{\prime}\right)^{k}$, then we define $\lceil x\rceil_{\epsilon^{\prime}}=\left(1+\epsilon^{\prime}\right)^{k},\lfloor x\rfloor_{\epsilon^{\prime}}=\left(1+\epsilon^{\prime}\right)^{k-1}$. If $x$ is an exact power of $\left(1+\epsilon^{\prime}\right)$, then $\lceil x\rceil_{\epsilon^{\prime}}=\lfloor x\rfloor_{\epsilon^{\prime}}=x$. Note that $\lceil x\rceil_{\epsilon^{\prime}} \leq\left(1+\epsilon^{\prime}\right) x$ for any $x \geq 1$.

For any $\epsilon>0$, we define the modified deteriorating rate of job $J_{j}$ as $b_{j}^{\prime}=\left\lceil 1+b_{j}\right\rceil_{\epsilon^{\prime}}-1$, the modified release date of job $J_{j}$ as $r_{j}^{\prime}=\left\lceil r_{j}\right\rceil_{\epsilon^{\prime}}$ and the modified due date of job $J_{j}$ as $d_{j}^{\prime}=(1+\epsilon) d_{j}$, where $\epsilon^{\prime}=\epsilon / 2(n+1)$. Let $L_{j}$ denote the exponent of $1+b_{j}^{\prime}$; that is, $1+b_{j}^{\prime}=\left\lceil 1+b_{j}\right\rceil_{\epsilon^{\prime}}=\left(1+\epsilon^{\prime}\right)^{L_{j}}$; then $L_{j}=\log \left\lceil 1+b_{j}\right\rceil_{\epsilon^{\prime}} / \log \left(1+\epsilon^{\prime}\right)=O\left(n \log \left(1+b_{j}\right) / \epsilon\right)$; let $l_{j}$ denote the exponent of $r_{j}^{\prime}$; that is, $r_{j}^{\prime}=\left\lceil r_{j}\right\rceil_{\epsilon^{\prime}}=\left(1+\epsilon^{\prime}\right)^{l_{j}}$; then $l_{j}=\log \left\lceil r_{j}\right\rceil_{\epsilon^{\prime}} / \log \left(1+\epsilon^{\prime}\right)=O\left(n \log r_{j} / \epsilon\right)$.

For any $\epsilon>0$, let $\epsilon^{\prime}=\epsilon / 2(n+1)$ and $R=\left\{J_{i_{1}}, J_{i_{2}}, \ldots, J_{i_{n}}\right\}$ be the set of the rejected jobs, where $i_{1}<i_{2}<\cdots<i_{m}$. The inflated rejection penalty of the set $R$ is defined as

$$
\phi_{\epsilon^{\prime}}(R)= \begin{cases}{\left[e_{i_{m}}+\phi_{\epsilon^{\prime}}\left(R-J_{i_{m}}\right)\right]_{\epsilon^{\prime}},} & \text { if } m \geq 1, \\ 0, & \text { if } R=\varnothing .\end{cases}
$$

Lemma 3 (see [11]). For any set $R$ of jobs and any $\epsilon^{\prime}>0$, $\phi_{\epsilon^{\prime}}(R) \leq\left(1+\epsilon^{\prime}\right)^{|R|} \sum_{j_{j} \in R} e_{j}$.

Lemma 4 (see [19]). For any $0<x \leq 1$ and for any integer $n \geq 1,(1+x / n)^{n} \leq 1+2 x$ holds.
Theorem 5. For any $0<\epsilon \leq 2$, the optimal objective function value for $1 \mid r_{j}, p_{j}=b_{j} t$, rej, agreeable $\mid L_{\max }(\bar{R})+\phi_{\epsilon^{\prime}}(R)$ with the modified deteriorating rates, the modified release dates, and the modified due dates is at most $(1+\epsilon)$ times the optimal objective function value for $1 \mid r_{j}, p_{j}=b_{j} t$, rej, agreeable | $L_{\max }(\bar{R})+\sum_{J_{j} \in R} e_{j}$.

Proof. Assume that jobs in the set $\bar{R}$ are scheduled in the order of $[1], \ldots,[|\bar{R}|]$. Let $C_{j}^{\prime}$ denote the completion time of job $J_{j}$ under the modified deteriorating rates and release dates; we have $C_{j}^{\prime}=\max _{1 \leq i \leq j}\left\{r_{[i]}^{\prime} \prod_{k=i}^{j}\left(1+b_{[k]}^{\prime}\right)\right\}$, then

$$
\begin{aligned}
L_{j}^{\prime} & =C_{j}^{\prime}-d_{j}^{\prime}=\max _{1 \leq i \leq j}\left\{r_{[i]}^{\prime} \prod_{k=i}^{j}\left(1+b_{[k]}^{\prime}\right)\right\}-d_{[j]}^{\prime} \\
& \leq\left(1+\epsilon^{\prime}\right)^{j+1} \max _{1 \leq i \leq j}\left\{r_{[i]} \prod_{k=i}^{j}\left(1+b_{[k]}\right)\right\}-(1+\epsilon) d_{[j]} \\
& \leq\left(1+\epsilon^{\prime}\right)^{n+1} C_{j}-(1+\epsilon) d_{j} \\
& \leq(1+\epsilon) L_{j} .
\end{aligned}
$$

The objective function is

$$
\begin{aligned}
L_{\max }^{\prime}(\bar{R})+\phi_{\epsilon^{\prime}}(R) & \leq \max \left\{L_{j}^{\prime}\right\}+\left(1+\epsilon^{\prime}\right)^{|R|} \sum_{J_{j} \in R} e_{j} \\
& \leq(1+\epsilon)\left(L_{\max }(\bar{R})+\sum_{J_{j} \in R} e_{j}\right) .
\end{aligned}
$$

Since the above inequality is valid for any $\bar{R} \subseteq \mathscr{J}$, the results hold.

Assume that the jobs have been reindexed such that $d_{1} \leq$ $\cdots \leq d_{n}$ and define

$$
\tau_{k}= \begin{cases}\left(1+\epsilon^{\prime}\right)^{k}, & \text { if } k \neq-1, \\ 0, & \text { if } k=-1 .\end{cases}
$$

Let $f(j, \lambda, k)$ denote the minimum value of the maximum lateness satisfying the following conditions.

(i) The jobs in consideration are $J_{1}, \ldots, J_{j}$.

(ii) The maximum completion time of the accepted jobs is $\left(1+\epsilon^{\prime}\right)^{\lambda}$.

(iii) The inflated rejection penalty of the rejected jobs is $\tau_{k}$.

In any such schedule, there are two possible cases: either job $J_{j}$ is rejected or $J_{j}$ is accepted and processed on the machine.

Case 1 (Job $J_{j}$ is rejected). Let $\left(1+\epsilon^{\prime}\right)^{k^{\prime}}$ be the inflated rejection penalty of the rejected jobs among $J_{1}, \ldots, J_{j-1}$. From the definition of inflated rejection penalty, the largest value of $k^{\prime}$ is given by $\left(1+\epsilon^{\prime}\right)^{\tilde{k}}=\left\lfloor\left(1+\epsilon^{\prime}\right)^{k}-e_{j}\right\rfloor_{\epsilon^{\prime}}$. Hence, the value of the maximum lateness for the optimal schedule is $\min _{-1 \leq k^{\prime} \leq \tilde{k}} f\left(j-1, \lambda, k^{\prime}\right)$. 
Case 2 (Job $J_{j}$ is accepted). Consider the following.

Case $2.1\left(\lambda>l_{j}+L_{j}\right)$. In this case, the maximum completion time of accepted jobs among $J_{1}, \ldots, J_{j-1}$ is $\left(1+\epsilon^{\prime}\right)^{\lambda} /\left(1+\epsilon^{\prime}\right)^{L_{j}}$. Therefore, $f(j, \lambda, k)=\max \left\{f\left(j-1, \lambda-L_{j}, k\right),\left(1+\epsilon^{\prime}\right)^{\lambda}-d_{j}^{\prime}\right\}$.

Case $2.2\left(\lambda=l_{j}+L_{j}\right)$. In this case, let $\left(1+\epsilon^{\prime}\right)^{\lambda^{\prime}}$ be the maximum completion time of accepted jobs among $J_{1}, \ldots, J_{j-1}$. Since the starting time of job $J_{j}$ is $r_{j}^{\prime}$, we have $\lambda^{\prime} \leq l_{j}$. Hence, $f(j, \lambda, k)=\max \left\{\min _{0 \leq \lambda^{\prime} \leq l_{j}} f\left(j-1, \lambda^{\prime}, k\right),(1+\right.$ $\left.\left.\epsilon^{\prime}\right)^{\lambda}-d_{j}^{\prime}\right\}$.

Combining the above two cases, the dynamic programming algorithm is stated as follows.

\section{Algorithm DP1}

Step 1 (initialization).

$$
f(1, \lambda, k)= \begin{cases}r_{1}^{\prime}\left\lceil 1+b_{1}\right\rceil_{\epsilon^{\prime}}-d^{\prime} & \lambda=l_{1}+L_{1}, \quad k=-1, \\ -\infty & \lambda=0, \tau_{k}=\left\lceil e_{1}\right\rceil_{\epsilon^{\prime}}, \\ +\infty & \text { otherwise. }\end{cases}
$$

Step 2 (recursion). If $\lambda<l_{j}+L_{j}, f(j, \lambda, k)=\min _{-1 \leq k^{\prime} \leq \tilde{k}} f(j-$ $\left.1, \lambda, k^{\prime}\right)$,

If $\lambda>l_{j}+L_{j}$,

$$
\begin{gathered}
f(j, \lambda, k) \\
=\min \left\{\max \left\{f\left(j-1, \lambda-L_{j}, k\right),\left(1+\epsilon^{\prime}\right)^{\lambda}-d_{j}^{\prime}\right\},\right. \\
\left.\min _{-1 \leq k^{\prime} \leq k} f\left(j-1, \lambda, k^{\prime}\right)\right\} .
\end{gathered}
$$

$$
\begin{aligned}
& \text { If } \lambda=l_{j}+L_{j}, \\
& f(j, \lambda, k) \\
& =\min \left\{\max \left\{\min _{0 \leq \lambda^{\prime} \leq l_{j}} f\left(j-1, \lambda^{\prime}, k\right),\left(1+\epsilon^{\prime}\right)^{\lambda}-d_{j}^{\prime}\right\},\right. \\
& \left.\min _{-1 \leq k^{\prime} \leq \widehat{k}} f\left(j-1, \lambda, k^{\prime}\right)\right\},
\end{aligned}
$$

where $\widetilde{k}$ is given by $\left(1+\epsilon^{\prime}\right)^{\widetilde{k}}=\left\lfloor\left(1+\epsilon^{\prime}\right)^{k}-e_{j}\right\rfloor_{\epsilon^{\prime}}$.

Step 3 (output). The optimal value is determined by $\min \left\{f(n, \lambda, k)+\tau_{k}: 0 \leq \lambda \leq \max _{1 \leq i \leq n}\left\{l_{i}\right\}+\sum_{j=1}^{n} L_{j},-1 \leq\right.$ $k \leq K\}$, where $K$ is the smallest integer such that $\left(1+\epsilon^{\prime}\right)^{K} \geq\left(1+\epsilon^{\prime}\right)^{n} \sum_{j=1}^{n} e_{j}$.

Let $L=\log \max \left\{r_{\max }, 1+b_{\max }, \sum_{j=1}^{n} e_{j}\right\}$, where $r_{\max }=$ $\max _{1 \leq j \leq n} r_{j}, b_{\max }=\max _{1 \leq j \leq n} b_{j}$. We have the following theorem.

Theorem 6. There exists an FPTAS for problem $1 \mid r_{j}, p_{j}=$ $b_{j} t$, rej, agreeable | $L_{\max }(\bar{R})+\sum_{J_{j} \in R} e_{j}$ which runs in $O\left(n^{7} L^{4} / \epsilon^{4}\right)$ time.
Proof. We need to compute exactly $O\left(n\left(\max _{1 \leq i \leq n}\left\{l_{i}\right\}+\right.\right.$ $\left.\left.\sum_{j=1}^{n} L_{j}+1\right)(K+2)\right)$ values $f(j, \lambda, k)$ and computation of each value takes at most $O\left(\left(\max _{1 \leq i \leq n}\left\{l_{i}\right\}+\sum_{j=1}^{n} L_{j}\right) K\right)$ time, where $K=O\left((n / \epsilon) \log \sum_{j=1}^{n} e_{j}\right)$. Hence, the running time of Algorithm DP1 is

$$
\begin{aligned}
& O\left(n\left(\max _{1 \leq i \leq n}\left\{l_{i}\right\}+\sum_{j=1}^{n} L_{j}\right)^{2} K^{2}\right) \\
& =O\left(n\left(\frac{n \log r_{\max }}{\epsilon}+\frac{n^{2} \log \left(1+b_{\max }\right)}{\epsilon}\right)^{2}\right. \\
& \left.\quad \times\left(\frac{n \log \sum_{j=1}^{n} e_{j}}{\epsilon}\right)^{2}\right) \\
& =O\left(\frac{n^{7} L^{4}}{\epsilon^{4}}\right) .
\end{aligned}
$$

\section{Parallel-Machine Problem}

4.1. An FPTAS. In this subsection, we focus our attention on identical parallel machines problem with deterioration and rejection. For convenience of discussion, we consider a scheduling model in which each job $J_{j}$ has a delivery time $q_{j} \geq 0$ rather than a due date $d_{j}$. When all jobs have identical release dates, the problem $P m \mid r_{j}=t_{0}, p_{j}=$ $b_{j} t$, rej | $D_{\max }(\bar{R})+\sum_{J_{j} \in R} e_{j}$ is at least ordinarily NP-hard according to Kononov [20]. We give a fully polynomial time approximation scheme to solve it by considering the modified deteriorating rates, the modified delivery times, and the inflated rejection penalty.

For any $\epsilon>0$, we define the modified deteriorating rate and delivery time of job $J_{j}$ as $b_{j}^{\prime}=\left\lceil 1+b_{j}\right\rceil_{\epsilon^{\prime}}-1, q_{j^{\prime}}=\left(1+\epsilon^{\prime}\right) q_{j}$, where $\epsilon^{\prime}=\epsilon / 2 n$. Let $L_{j}$ denote the exponent of $1+b_{j}^{\prime}$; that is, $1+b_{j}^{\prime}=\left\lceil 1+b_{j}\right\rceil_{\epsilon^{\prime}}=\left(1+\epsilon^{\prime}\right)^{L_{j}}$; then $L_{j}=\log \left\lceil 1+b_{j}\right\rceil_{\epsilon^{\prime}} / \log (1+$ $\left.\epsilon^{\prime}\right)=O\left(n \log \left(1+b_{j}\right) / \epsilon\right)$.

For any $\epsilon>0$, let $\epsilon^{\prime}=\epsilon / 2 n$ and $R=\left\{J_{i_{1}}, J_{i_{2}}, \ldots, J_{i_{m}}\right\}$ be the set of the rejected jobs, where $i_{1}<i_{2}<\cdots<i_{m}$. The inflated rejection penalty of the set $R$ is defined as

$$
\phi_{\epsilon^{\prime}}(R)= \begin{cases}{\left[e_{i_{m}}+\phi_{\epsilon^{\prime}}\left(R-J_{i_{m}}\right)\right]_{\epsilon^{\prime}},} & \text { if } m \geq 1, \\ 0, & \text { if } R=\varnothing .\end{cases}
$$

Lemma 7. For any $0<\epsilon \leq 2$, the optimal objective function value for $P m \mid r_{j}=t_{0}, p_{j}=b_{j} t$, rej $\mid D_{\max }(\bar{R})+\phi_{\epsilon^{\prime}}(R)$ with the modified deteriorating rates and the modified delivery times is at most $(1+\epsilon)$ times the optimal objective function value for

$$
P m\left|r_{j}=t_{0}, p_{j}=b_{j} t, r e j\right| D_{\max }(\bar{R})+\sum_{J_{j} \in R} e_{j} .
$$

Lemma 8. There exists an optimal job sequence for $P m \mid r_{j}=$ $t_{0}, p_{j}=b_{j} t$, rej $\mid D_{\max }(\bar{R})+\sum_{J_{j} \in R} e_{j}$ such that on each machine the accepted jobs are sequenced in nonincreasing order of $q_{j}$. 
Based on Lemmas 7 and 8, we design a dynamic programming algorithm for $P m \mid r_{j}=t_{0}, p_{j}=b_{j} t$, rej | $D_{\max }(\bar{R})+\phi_{\epsilon^{\prime}}(R)$ with the modified deteriorating rates and delivery times. Let us index the jobs such that $q_{1} \geq \cdots \geq q_{n}$ and define

$$
\tau_{k}= \begin{cases}\left(1+\epsilon^{\prime}\right)^{k}, & \text { if } k \neq-1 \\ 0, & \text { if } k=-1\end{cases}
$$

Let $F\left(j, \lambda_{1}, \ldots, \lambda_{m}, k\right)$ denote the optimal value of the maximum delivery completion time satisfying the following conditions.

(i) The jobs in consideration are $J_{1}, \ldots, J_{j}$.

(ii) The maximum completion time of the accepted jobs on machine $M_{i}$ is $t_{0}\left(1+\epsilon^{\prime}\right)^{\lambda_{i}}$ for $i=1, \ldots, m$.

(iii) The inflated rejection penalty of the rejected jobs is $\tau_{k}$.

The dynamic programming algorithm is stated as follows.

\section{Algorithm DP2}

Step 1 (initialization).

$$
\begin{aligned}
& F\left(1, \lambda_{1}, \ldots, \lambda_{m}, k\right) \\
& = \begin{cases}t_{0}\left(1+\epsilon^{\prime}\right)^{L_{1}}+q_{1}^{\prime}, & \left(\lambda_{1}, \ldots, \lambda_{i}, \ldots, \lambda_{m}, k\right) \\
& =\left(0, \ldots, L_{1}, \ldots, 0,-1\right), \\
-\infty, & \left(\lambda_{1}, \ldots, \lambda_{i}, \ldots, \lambda_{m}\right) \\
& =(0, \ldots, 0, \ldots, 0), \\
& \\
& \begin{array}{l}
\tau_{k}=\left\lceil e_{1}\right\rceil_{\epsilon^{\prime}} \\
\text { otherwise. }
\end{array}\end{cases}
\end{aligned}
$$

Step 2 (recursion). If job $J_{j}$ is rejected,

$$
F\left(j, \lambda_{1}, \ldots, \lambda_{m}, k\right)=\min _{-1 \leq k^{\prime} \leq \tilde{k}} F\left(j-1, \lambda_{1}, \ldots, \lambda_{m}, k^{\prime}\right) .
$$

If job $J_{j}$ is accepted,

$$
\begin{gathered}
F\left(j, \lambda_{1}, \ldots, \lambda_{m}, k\right) \\
=\min _{1 \leq i \leq m}\left\{\operatorname { m a x } \left\{F\left(j-1, \lambda_{1}, \ldots, \lambda_{i}-L_{i}, \ldots, \lambda_{m}, k\right),\right.\right. \\
\left.\left.t_{0}\left(1+\epsilon^{\prime}\right)^{\lambda_{i}}+q_{j}^{\prime}\right\}\right\} .
\end{gathered}
$$

Combining the two cases, we have

$$
\begin{gathered}
F\left(j, \lambda_{1}, \ldots, \lambda_{m}, k\right) \\
=\min \left\{\operatorname { m i n } _ { 1 \leq i \leq m } \left\{\operatorname { m a x } \left\{F\left(j-1, \lambda_{1}, \ldots, \lambda_{i}-L_{i}, \ldots, \lambda_{m}, k\right),\right.\right.\right. \\
\left.\left.t_{0}\left(1+\epsilon^{\prime}\right)^{\lambda_{i}}+q_{j}^{\prime}\right\}\right\}, \\
\left.\min _{-1 \leq k^{\prime} \leq \tilde{k}} F\left(j-1, \lambda_{1}, \ldots, \lambda_{m}, k^{\prime}\right)\right\},
\end{gathered}
$$

where $\tilde{k}$ is given by $\left(1+\epsilon^{\prime}\right)^{\widetilde{k}}=\left\lfloor\left(1+\epsilon^{\prime}\right)^{k}-e_{j}\right\rfloor_{\epsilon^{\prime}}$.
Step 3 (output). The optimal value is determined by

$$
\begin{gathered}
\min \left\{F\left(n, \lambda_{1}, \ldots, \lambda_{m}, k\right)+\tau_{k}: 0 \leq \lambda_{i} \leq \sum_{j=1}^{n} L_{j},\right. \\
1 \leq i \leq m,-1 \leq k \leq K\},
\end{gathered}
$$

where $K$ is the smallest integer such that $\left(1+\epsilon^{\prime}\right)^{K} \geq(1+$ $\left.\epsilon^{\prime}\right)^{n} \sum_{j=1}^{n} e_{j}$.

Theorem 9. There exists an FPTAS for problem $P m \mid r_{j}=$ $t_{0}, p_{j}=b_{j} t$, rej $\mid D_{\max }(\bar{R})+\sum_{J_{j} \in R} e_{j}$ which runs in $O\left(\left(n^{m+3} / \epsilon^{m+2}\right)\left(\sum_{j=1}^{n} \log \left(1+b_{j}\right)\right)^{m} \log ^{2}\left(\sum_{j=1}^{n} e_{j}\right)\right)$ time.

Proof. We need to compute $n\left(\sum_{j=1}^{n} L_{j}+1\right)^{m}(K+2)$ values $F\left(j, \lambda_{1}, \ldots, \lambda_{m}, k\right)$. Computation of each such value takes $O(K)$ time, where $K=O\left((n / \epsilon) \log \sum_{j=1}^{n} e_{j}\right)$. Therefore the overall time of the algorithm is $O\left(n\left(\sum_{j=1}^{n} L_{j}\right)^{m} K^{2}\right)=$ $O\left(\left(n^{m+3} / \epsilon^{m+2}\right)\left(\sum_{j=1}^{n} \log \left(1+b_{j}\right)\right)^{m} \log ^{2}\left(\sum_{j=1}^{n} e_{j}\right)\right)$.

4.2. A Heuristic Algorithm for a Special Case. In this subsection, it is assumed that all jobs have to be processed; that is, rejection is not allowed. This problem can be denoted by $P m\left|r_{j}=t_{0}, p_{j}=b_{j} t\right| D_{\max }$. We present a heuristic algorithm and give its worst case analysis.

Heuristic DLS. Consider the following steps.

Step 1. Place all jobs in a list ordered by nonincreasing delivery times. Set $S_{i}=t_{0}$ for $1 \leq i \leq m$, and $S_{i}$ denotes the total completion time of jobs assigned to machine $M_{i}$.

Step 2. Select a machine $M_{i}$ for which $S_{i}$ is as small as possible. Choose the first unscheduled job $J_{j}$ of the list and assign it to machine $M_{i}$. Set $S_{i}=S_{i}\left(1+b_{j}\right)$.

Step 3. Repeat Step 2 until all jobs are scheduled.

Theorem 10. The worst case performance ratio of heuristic DLS is $\left(1+b_{\max }\right)^{1-1 / m}$ and this bound is tight.

Proof. Let $D_{\max }^{\mathrm{DLS}}$ be the maximum delivery completion time according to heuristic DLS and let $D_{\max }^{*}$ be the corresponding time in an optimal schedule. Assume that there exists a counterexample with $D_{\max }^{\mathrm{DLS}} / D_{\max }^{*}>\left(1+b_{\max }\right)^{1-1 / m}$. Let $J_{k}$ be the first job in the sorted list with $C_{k}+q_{k}>(1+$ $\left.b_{\max }\right)^{1-1 / m} D_{\max }^{*}$, remove all the following jobs from the list, and produce a minimum counterexample with $D_{\max }^{\mathrm{DLS}}=C_{k}+$ $q_{k}$, where $J_{k}$ is the job with smallest delivery time. Next, we transform the counterexample by subtracting $q_{k}$ from all delivery times. Obviously, this decreases both $D_{\max }^{\mathrm{DLS}}$ and $D_{\max }^{*}$ by $q_{k}$. Since

$$
\frac{D_{\max }^{\mathrm{DLS}}-q_{k}}{D_{\max }^{*}-q_{k}} \geq \frac{D_{\max }^{\mathrm{DLS}}}{D_{\max }^{*}}>\left(1+b_{\max }\right)^{1-1 / m}
$$


TABLE 1: Summary of the computational results.

\begin{tabular}{cccccc}
\hline$m$ & $n$ & $L\left(D_{\max }^{*}\right)$ & $D_{\max }^{\text {DLS }}$ & $\frac{D_{\max }^{\text {DLS }}}{L\left(D_{\max }^{*}\right)}$ & \multicolumn{2}{c}{$\frac{\left|D_{\max }^{\text {DLS }}-L\left(D_{\max }^{*}\right)\right|}{L\left(D_{\max }^{*}\right)}$} \\
\hline 3 & 10 & 240 & 559 & 2.329 & 1.329 \\
4 & 12 & 208 & 577 & 2.774 & 1.774 \\
4 & 12 & 168 & 485 & 2.887 & 1.887 \\
4 & 15 & 380 & 520 & 1.368 & 0.368 \\
4 & 15 & 477 & 931 & 1.952 & 0.952 \\
5 & 10 & 33 & 60 & 2.018 & 1.818 \\
5 & 10 & 57 & 115 & 2.762 & 1.018 \\
5 & 18 & 611 & 1688 & \\
\hline
\end{tabular}

holds, the transformation yields another counterexample. Now the last job has delivery times zero and $D_{\max }^{\mathrm{DLS}}=C_{k}$. At the time before job $J_{k}$ is assigned to its machine $M_{i}$,

$$
S_{i} \leq \frac{\left(\prod_{l=1}^{k}\left(1+b_{1}\right)\right)^{1 / m}}{\left(1+b_{k}\right)^{1 / m}} \leq \frac{D_{\max }^{*}}{\left(1+b_{k}\right)^{1 / m}}
$$

This implies

$$
\begin{aligned}
D_{\max }^{\mathrm{DLS}} & =C_{k}=S_{i}\left(1+b_{k}\right) \leq\left(1+b_{k}\right)^{1-1 / m} D_{\max }^{*} \\
& \leq\left(1+b_{\max }\right)^{1-1 / m} D_{\max }^{*} .
\end{aligned}
$$

To prove that the bound is tight, consider the following example with $n=m(m-1)+1$ jobs, all jobs have delivery time zero, the first $m(m-1)$ of them has deteriorating rates $b_{j}=1, j=1,2, \ldots, m(m-1)$, and the last job's deteriorating rate is $b_{n}=2^{m}-1$. Then $b_{\max }=b_{n}$ and $\left(1+b_{\max }\right)^{1-1 / m}=2^{m-1}$. By DLS, $D_{\max }^{\mathrm{DLS}}=t_{0}\left(1+b_{j}\right)^{m-1}\left(1+b_{n}\right)=t_{0} 2^{m-1} 2^{m}=t_{0} 2^{2 m-1}$. In an optimal schedule, $J_{n}$ is assigned to one machine and all the previous $m(m-1)$ jobs are equally assigned to the other $m-1$ machines. This yields to $D_{\max }^{*}=t_{0} 2^{m}$. Thus,

$$
\frac{D_{\max }^{\mathrm{DLS}}}{D_{\max }^{*}}=\left(1+b_{\max }\right)^{1-1 / m}=2^{m-1} \text {. }
$$

This completes the proof.

4.3. Numerical Experiment. This section conducts some numerical experiments to evaluate the performance of heuristic DLS. Without loss of generality, we set $t_{0}=1$. The delivery time $q_{j}, 1 \leq j \leq n$ and the deteriorating rate $b_{j}, 1 \leq j \leq n$ are generated from $U[0,50]$ and $U[1,10]$, respectively. The lower bound of optimal value is $L\left(D_{\max }^{*}\right)=$ $\left(\prod_{l=1}^{n}\left(1+b_{l}\right)\right)^{1 / m}$. The numerical results are summarized in Table 1. The program was coded in Matlab. The results of numerical experiments show that the algorithm has good performance. The proposed algorithm is acceptable for the NP-hard problem.

\section{Conclusion}

In this paper, we focus on several scheduling problems with deterioration, release date, and rejection. For the single machine model, we show that this problem is strongly NPhard and provides a fully polynomial time approximation scheme when all jobs have agreeable release dates and due dates. For parallel machines model, we propose a fully polynomial time approximation scheme for $P m \mid r_{j}=$ $t_{0}, p_{j}=b_{j} t$, rej $\mid D_{\max }(\bar{R})+\sum_{J_{j} \in R} e_{j}$ and a heuristic algorithm for a special case. The computational results show that the heuristics is efficient in obtaining near-optimal solutions. For future research, it would be interesting to focus on scheduling deteriorating jobs and rejection with other objectives. Furthermore, investigation of the online version of this scheduling model is also an interesting research direction.

\section{Conflict of Interests}

The authors declare that there is no conflict of interests regarding the publication of this paper.

\section{Acknowledgments}

This paper is supported by the National Natural Science Foundation of China (11201259), the Doctoral Fund of the Ministry of Education (20123705120001 and 20123705110003), and Promotive Research Fund for Young and Middle-aged Scientists of Shandong Province (BS2013SF016).

\section{References}

[1] S. Browne and U. Yechiali, "Scheduling deteriorating jobs on a single processor," Operations Research, vol. 38, no. 3, pp. 495498, 1990.

[2] G. Mosheiov, "Scheduling jobs under simple linear deterioration," Computers and Operations Research, vol. 21, no. 6, pp. 653659, 1994.

[3] B. Alidaee and N. K. Womer, "Scheduling with time dependent processing times: review and extensions," Journal of the Operational Research Society, vol. 50, no. 7, pp. 711-720, 1999.

[4] T. C. E. Cheng, Q. Ding, and B. M. T. Lin, "A concise survey of scheduling with time-dependent processing times," European Journal of Operational Research, vol. 152, no. 1, pp. 1-13, 2004.

[5] P. Shen, C. M. Wei, and X. Huang, "Single-machine scheduling problems with an actual time-dependent deterioration," Applied 
Mathematical Modelling. Simulation and Computation for Engineering and Environmental Systems, vol. 37, no. 7, pp. 5555-5562, 2013.

[6] C. Miao, Y. Zhang, and C. Wu, "Scheduling of deteriorating jobs with release dates to minimize the maximum lateness," Theoretical Computer Science, vol. 462, pp. 80-87, 2012.

[7] P. Liu, N. Yi, X. Zhou, and H. Gong, "Scheduling two agents with sum-of-processing-times-based deterioration on a single machine," Applied Mathematics and Computation, vol. 219, no. 17, pp. 8848-8855, 2013.

[8] Y. Bartal, S. Leonardi, A. Marchetti-Spaccamela, J. Sgall, and L. Stougie, "Multiprocessor scheduling with rejection," SIAM Journal on Discrete Mathematics, vol. 13, no. 1, pp. 64-78, 2000.

[9] H. Hoogeveen, M. Skutella, and G. J. Woeginger, "Preemptive scheduling with rejection," Mathematical Programming, vol. 94, no. 2-3, pp. 361-374, 2003.

[10] L. Zhang, L. Lu, and J. Yuan, "Single machine scheduling with release dates and rejection," European Journal of Operational Research, vol. 198, no. 3, pp. 975-978, 2009.

[11] S. Sengupta, "Algorithms and approximation schemes for minimum lateness/tardiness scheduling with rejection," in Algorithms and Data Structures, vol. 2748 of Lecture Notes in Computer Science, pp. 79-90, Springer, Berlin, Germany, 2003.

[12] D. Shabtay, N. Gaspar, and M. Kaspi, "A survey on offline scheduling with rejection," Journal of Scheduling, vol. 16, no. 1, pp. 3-28, 2013.

[13] Y. Cheng and S. Sun, "Scheduling linear deteriorating jobs with rejection on a single machine," European Journal of Operational Research, vol. 194, no. 1, pp. 18-27, 2009.

[14] S. Li and J. Yuan, "Parallel-machine scheduling with deteriorating jobs and rejection," Theoretical Computer Science, vol. 411, no. 40-42, pp. 3642-3650, 2010.

[15] C. J. Hsua and C. W. Chang, "Unrelated parallel-machine scheduling with deteriorating jobs and rejection," Applied Mechanics and Materials, vol. 263-266, no. 1, pp. 655-659, 2013.

[16] R. L. Graham, E. L. Lawler, J. K. Lenstra, and A. H. G. Rinnooy Kan, "Optimization and approximation in deterministic sequencing and scheduling: a survey," Annals of Discrete Mathematics, vol. 5, pp. 287-326, 1979.

[17] M. R. Garey and D. S. Johnson, Computers and Intractability: A Guide to the Theory of NP-Completeness, W. H. Freeman, New York, NY, USA, 1979.

[18] F. Afrati, E. Bampis, C. Chekuri et al., "Approximation schemes for minimizing average weighted completion time with release dates," in Proceedings of the 40th Annual IEEE Symposium on Foundations of Computer Science, pp. 32-44, New York, NY, USA, 1999.

[19] D. W. Engels, D. R. Karger, S. G. Kolliopoulos, S. Sengupta, R. N. Uma, and J. Wein, "Techniques for scheduling with rejection," Journal of Algorithms. Cognition, Informatics and Logic, vol. 49, no. 1, pp. 175-191, 2003.

[20] A. Kononov, "Scheduling problems with linear increasing processing times," in Operations Research Proceedings 1996, pp. 208-212, Springer, Berlin, Germany, 1997. 


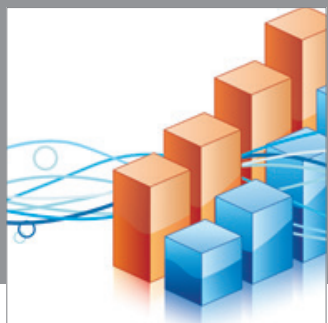

Advances in

Operations Research

mansans

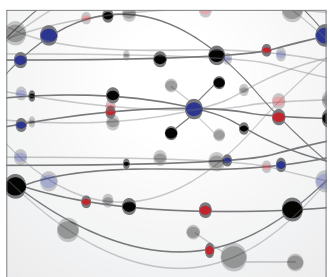

The Scientific World Journal
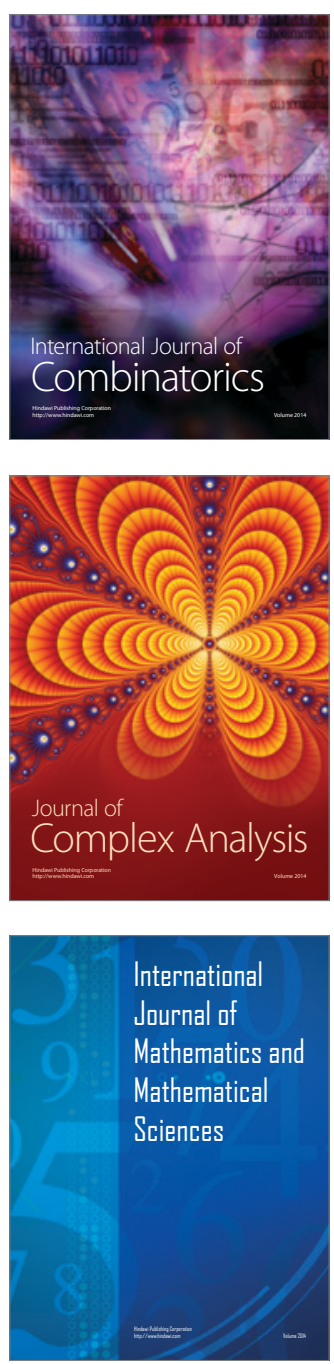
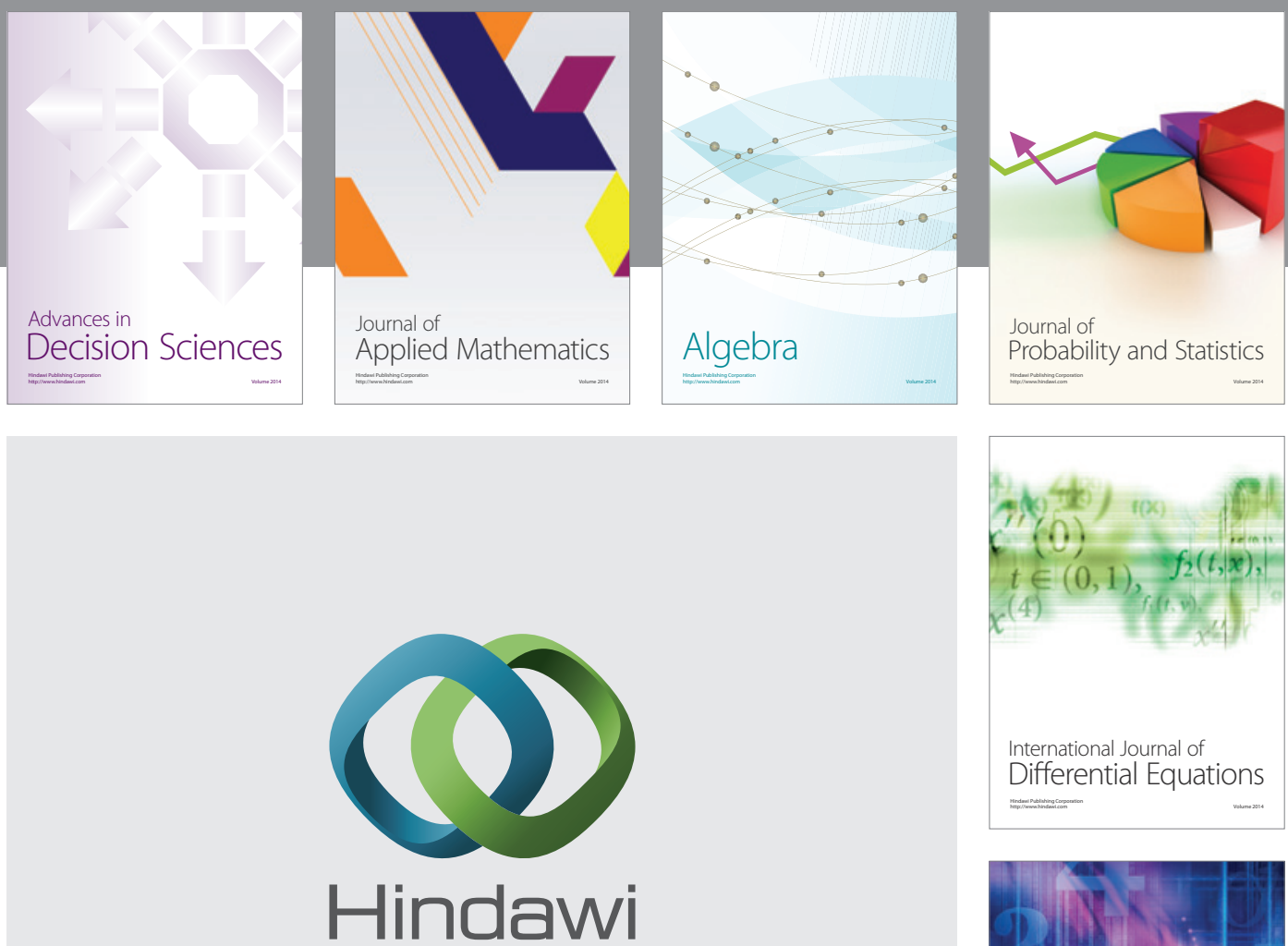

Submit your manuscripts at http://www.hindawi.com
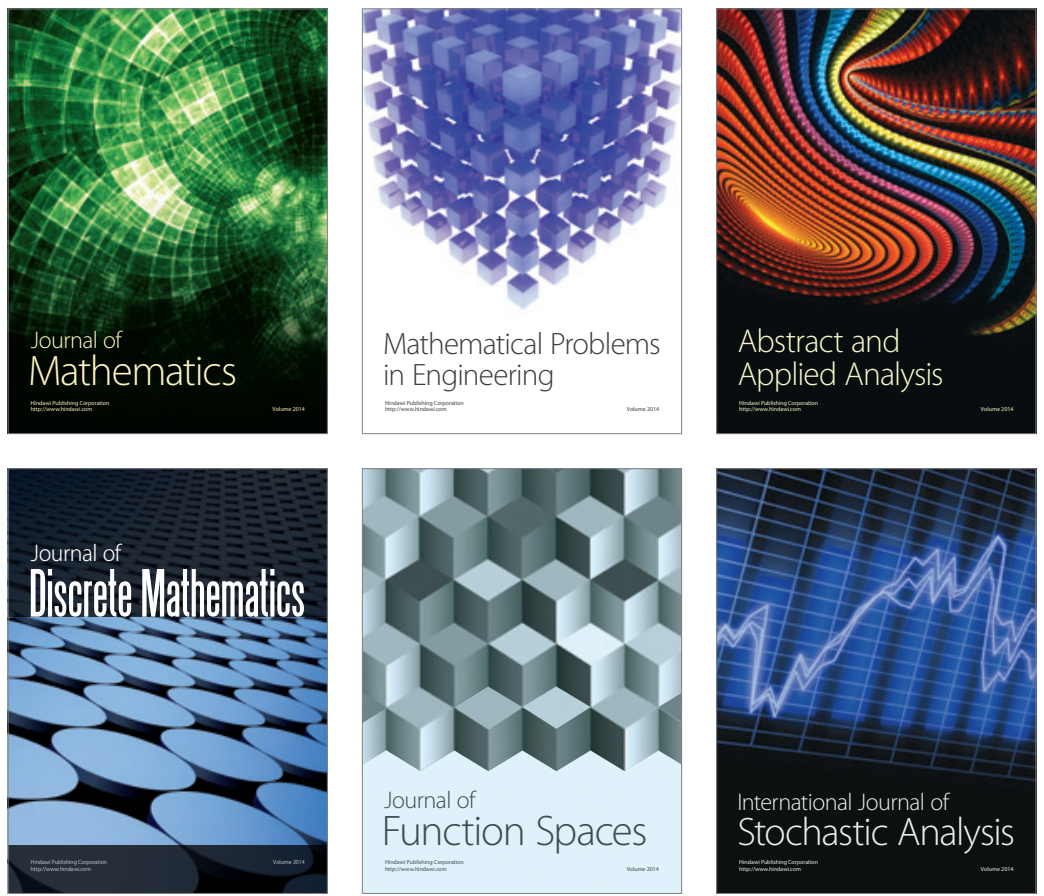

Journal of

Function Spaces

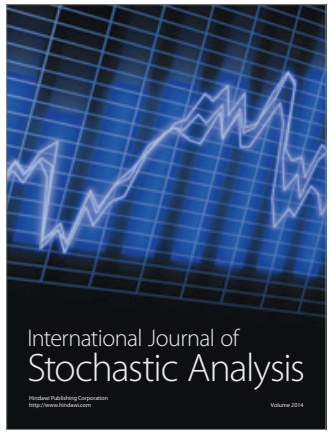

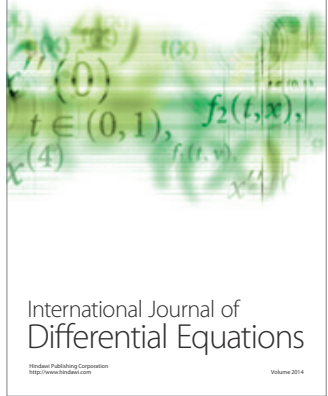
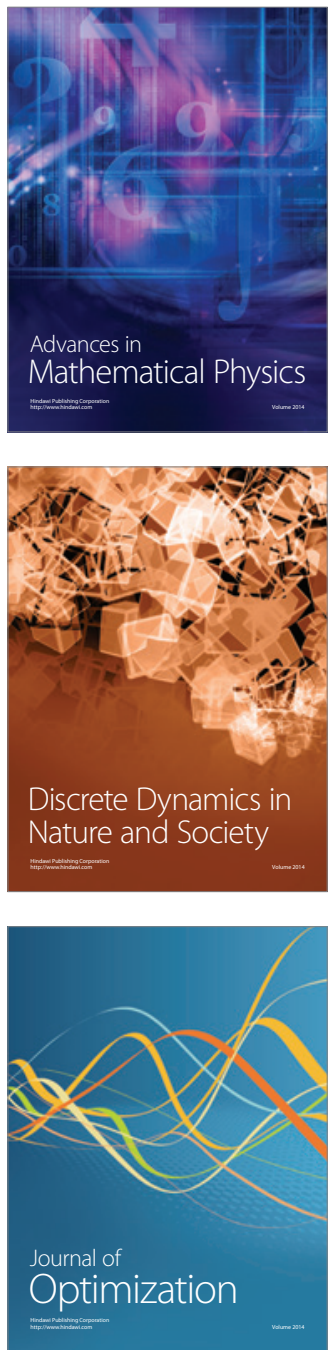